\title{
Organic wine production in Brazil: Challenges and limitations I La production de vin biologique au Brésil - défis et limitations
}

\author{
Marcos -Vinícius Araujo ${ }^{a}$, Kelly Lissandra Bruch, et Daniela Callegaro de Menezes \\ Centre of Studies and Researches in Agribusiness - Federal University of Rio Grande do Sul, Av. Bento Gonçalves, 7712 \\ Porto Alegre, RS, Brazil
}

\begin{abstract}
From the consumer pressure to healthier and sustainable food, and the search for quality of life, companies in various activities have reinvented to meet this demand. Organic products that have features to meet this trend, also took their place in the Brazilian wine industry, that is entered in this field to stay competitive. On the other hand, they found limiting factors that inhibiting the growth in supply of this product. In this perspective, the objective of this study is to describe and analyze what are the limiting factors for organic wine production in Brazil. To this end, an exploratory research was conducted from indepth interviews, secondary data and direct observation. The collected data were crossed between companies and theory. The results are presented in the following chain of organic wine, based on primary resources production - distribution - marketing and stakeholders. For conclusion, it is notable that wineries holding a major concern to maintain the certification, but the biggest challenge is to produce goodorganic grapes. On the other hand, it still requires marketing efforts, so that the product value can reach the end consumer

Résumé. A partir des pressions des consommateurs à des aliments plus sains et durables, ainsi que la recherche pour la qualité de vie, des entreprises de diverses activités se sont réinventées pour répondre à cette demande. Les produits biologiques, qui ont des caractéristiques pour répondre à cette tendance, ont pris leur place également dans le secteur vinicole brésilien, qu'est entré dans ce domaine pour rester compétitif. Par contre, ils ont trouvé des facteurs limitatifs qu'inhibent la croissance de l'offre de ce produit. Dans cette perspective, l'objectif de cette étude est de décrire et analyser quels sont ces facteurs limitatifs pour la production de vin biologique au Brésil. À cette fin, une recherche exploratoire a été menée à partir d'entretiens approfondis, des données secondaires et l'observation directe. Les données récoltées ont été croisées entre les entreprises et la théorie. Les résultats sont présentés en suivant la chaine du vin biologique, basé sur les ressources primaires - fabrication - distribution - marketing et les parties prenantes. Par conclusion, il est notable que les établissements vinicoles maintien une préoccupation majeure pour maintenir la certification, mais que le défi le plus grand est de produire de bons raisins biologiques. D'autre côté, il faut encore des efforts marketing, de sorte que la valeur du produit puisse atteindre le consommateur final.
\end{abstract}

\section{Introduction}

Les consommateurs sont de plus en plus préoccupés par la qualité des aliments consommés, l'origine, les matières premières utilisées ainsi que leurs impacts environnementaux et sociaux. Dans ce contexte, les entreprises se tournent vers une production plus durable afin de minimiser ces impacts.

$\mathrm{Ce}$ changement est vu dans la prédisposition du consommateur pour des aliments durables, sains, nonartificiels ainsi que sur des aspects visuels [1]. Cette conscience sur l'environnement génère une pression du consommateur vers les attitudes des entreprises. Le consommateur qui est toujours plus exigeant et conscient de l'impact de ses actions, adoptent un mode de consommation plus responsable, car ils portent de plus en plus d'informations qui vont collaborer dans la réflexion sur les problématiques sociales et environnementales [2], ces changements sont nommés de politisation de la consommation [3].

En réponse à cela, les produits biologiques ont des caractéristiques qui satisfont cette tendance mondiale, vu leurs valeurs sociales et environnementales. Dans cette optique, les établissements vinicoles brésiliens ont aperçus cette conduction du marché vers la consommation durable et s'est adapté pour fournir des produit pour y répondre. De cette façon, les entreprises se sont approprié de cette forme de production pour en rester compétitif.

L'État de Rio Grande do Sul, dans la Région Sud du Brésil, est le principal producteur brésilien de raisins, principalement la « Serra Gaúcha 》 [4], en 2003 cela représentait $93 \%$ de toute la production brésilienne [5]. C'est la région $*$ pionnière dans la production de raisin biologique pour la production de vin, en 1997 [6].

Le premier producteur de raisin biologique pour la production de vin a débuté dans ce domaine à cause de ses inquiétudes * environnementales, vu qu' aujourd'hui ils utilisent encore beaucoup de produits chimiques pour la production de raisin, à cause des problèmes climatiques et d'humidité de la région, que ne sont pas si favorables à production de raisin.

Par la suite, d'autres vignobles ont ressentis aussi la pression des consommateurs pour des aliments plus sains,

\footnotetext{
a Auteur de Correspondance : araujomacros@gmail.com
}

(c) The Authors, published by EDP Sciences. This is an Open Access article distributed under the terms of the Creative Commons Attribution License 4.0 (http://creativecommons.org/licenses/by/4.0/). 
plus durables et les actions visant le bien-être et la qualité de vie, ce qui obligeât différentes entreprises à prendre en compte cette demande de plus en plus forte.

Toutefois, certains établissements ont trouvé des facteurs limitant qui inhibent la croissance de l'offre de vin biologique. Dans cette perspective, l'objectif de cette étude est de décrire et d'analyser quels sont ces facteurs limitant pour la production du vin biologique par les secteurs vinicoles brésilien.

\subsection{La production biologique}

La production biologique des aliments représentent pour les consommateurs un moment de dégustation plaisant et agréable [7]. D'autres attributs présentés par Lee et Yun [7] sont qu'ils trouvent des caractéristiques sensorielles, une valeur nutritive, un bien-être écologique, ainsi que la valeur de la crédibilité de ces produits. La demande croissante pour des produits biologiques sont données par l'inquiétude du consommateur et aussi l'offre du produit dans le marché et par la disponibilité dans les magasins [8].

Alors que pour être considéré biologique, il faut que le produit suive une réglementation, de manières qu'au Brésil est considéré biologique les produit dont la production respecte les ressources naturelles et socio-économiques en considérant l'intégrité culturelle des communautés rurales, utilisant des méthodes culturelles, manque un mot ici biologique en opposition aux produits synthétiques (agrochimie et produit phytosanitaire) et les organismes génétiquement modifiés, quel que soit le niveau du processus de production, de traitement, de stockage, ou de distribution et commercialisation [9].

De ce point de vue, pour être un produit biologique, il faut re-penser toute la filière productive, pour Porter (1989) y compris par toutes les activités qu'ajoutent de la valeur au produit. D'un autre côté, ceci comprend aussi une filière pour les matières premières, les fournisseurs, la fabrication (traitement), la distribution, commercialisation (points de ventes) et les consommateurs [11]. En effet, pour analyser les défis et les limitations dans la production biologique de vin au Brésil, on va utiliser l'ordre suivant : matières premières et fournisseurs - processus de production distribution et commercialisation - consommation.

\section{Matériel et méthode}

Pour répondre à cet objectif, une enquête exploratoire a été menée à partir d'entretiens approfondis, de données secondaires et d'observations directes. Les critères de sélection des établissements vinicoles étaient : (1) être certifiés par l'audit, en suivant la loi de l'agriculture biologique brésilienne; et (2) produire du vin et du jus de raisin biologique. D'après ces critères, le premier établissement vinicole qui a produit du vin biologique au Brésil a été pris en compte, pour illustrer la difficulté de la production de vin à partir de raisin vitis vinifera, les raisons qui ont faits qu'il a arrêté la production de vin biologique. L'analyse portera sur l'ensemble des données collecté dans les entreprises, pour ne pas les identifier.

Les données collectées dans les établissements vinicoles ont été analysées à la lumière de la théorie des chaînes (filières), par l'analyse de contenu simple, en croisant les données collectées entre répondants et la théorie de base. Les résultats sont présentés en suivant la chaîne du vin biologique, basé sur les ressources primaires - la fabrication - la distribution - la commercialisation et les parties prenantes.

\subsection{L'échantillon de recherche}

Cinq entreprises ont été interviewées, les trois premières entre mai et juillet 2015, et les deux dernières en août 2016. Par ordre de visite et d'interview, l'entreprise 1 est une cave à vin fin située à Caxias do Sul - RS, elle était la première à cultiver des raisins biologiques au Brésil, ils ont arrêté d'être certifié en 2007.

L'entreprise 2 est une Coopérative Vinicole situé à Bento Gonçalves-RS, elle a une gamme complète de produit et produisait du vin de table et du jus de raisin biologique, mais elle vient d'arrêter la commercialisation de produit biologique dans ce portefeuille.

L'entreprise 3 est aussi une Coopérative Vinicole, située à Garibaldi-RS et elle a une gamme complète des produits dérivés du raisin. Elle a été la première productrice de vin de table et jus de raisin biologique - 2001. Elle est aujourd'hui la plus grande productrice vinicole du Brésil.

L'entreprise 4 est un petit établissement vinicole situé à Bento Gonçalves-RS, il commercialise ses produits dans les grands réseaux de distribution au Brésil, il ne produit pas une gamme complète (vin fin et de table, rouge et blanc) mais leur production est concentrée dans le jus de raisin biologique ainsi que conventionnel.

L'entreprise 5 est un grand établissement vinicole situé à São Marcos-RS. Il a une gamme complète de produit et a été le premier vignoble à produire du jus de raisin « fortifié» c'est-à-dire, un produit plus riche en fibres alimentaires pensé pour un public plus exigent sur la qualité alimentaire.

\section{Résultats}

Au-delà des questions environnementales, les motivations de l'établissement 1 pour commencer à produire du vin biologique, après quelques années, d'autres vignobles ont vu les produits biologiques comme une opportunité de marché et ont profité de leurs structures productives pour produire du vin biologique, toutefois de vin de table, fabriqué à partir de raisins de cépages américains.

Toutefois, l'établissement 1 a décidé en 2007 d'abandonner la certification biologique, cependant il a continué à suivre une production « naturel » nom qu'il a donnée à la production biologique sans la certification. Pour autant, il a trouvé beaucoup de difficultés dans le processus, comme le prix de la certification biologique et l'acceptation du marché au prix du vin en comparaison des autres produits de la boutique.

En tout cas, d'autres établissement ont aussi commencé à produire du raisin biologique, initialement pour la production $\mathrm{du}$ vin, ensuite plus fortement pour la production de jus de raisin biologique, car les cépages américains, comme l'isabelle, a une qualité gustative très appréciée pour le jus. Tous les établissements ont relaté des difficultés dans le processus, ce qu'a fait qu'ils se concentraient leur production dans le jus et aussi repenser la façon de produire. Dans ce contexte-là, l'analyse se portera sur les étapes de la chaine productive. 


\subsection{Motivations}

Un élément important pour analyser les défis de la production du vin biologique dans au Brésil, ce sont les motivations, suivie de la tendance pour des produits durables, qu'est aperçu l'intensification à la fin du siècle dénier [12].

Cette tendance a été aperçue par les établissements, sauf l'établissement 1 et le 3 qu'ont eu des motivations internes, comme la décision de la direction et dans le cas 3 , la pression de la part des producteurs de raisin, associés à la coopérative, qui ont décidé de migrer leur production vers le système biologique, ce qu'a forcé la coopérative à traiter ces raisins.

Certains établissements vinicoles ont suivies par cette tendance mondiale à la recherche de produits sains et à faible impact pour l'environnement [13]. L'établissement 4 et 5 ont adopté cette positionnement. L'établissement 4 rapporte qu'aujourd'hui ses produits, même les conventionnelles, sont généralement considérés comme durable par les consommateurs qui visitent l'entreprise, à cause de son positionnement vers la production biologique.

D'autre part, l'établissement 5 a adopté d'autres produits sains, au-delà des habitudes dans le processus d'autres produits qui répondent à cette perspective, comme les aliments fonctionnels. L'établissement 2, a cherché à répondre que la demande et compléter sa gamme de produit, cependant, vu l'absence de perception de la valeur sur ce produit, cela l'a fait arrêter d'y commercialiser, étant donné qu'ils ne produisaient plus de vin biologique en interne dans l'entreprise, le produit était fabriqué par un tiers et commercialisée avec son étiquette.

\subsection{Ressources primaires et fournisseurs}

Cela a été l'étape qu'ils ont eue la plus grande difficulté et celle que présente plus limitations à la production. La région de la «Serra Gaucha », où est situé les établissements étudiés, en dépit d'être la plus grande productrice brésilienne de raisin pour traitementindustrialisation, y a encore de difficulté à produire cela. Les producteurs de cette région ont encore besoin de l'utilisation intensif de produits agrochimiques. Cela a été rapporté par tous les établissements vinicoles, étant donnée l'établissement 4 présente les nouvelles frontières vinicoles du pays comme l'alternative, comme la région de la « Campanha Gaúcha, » à l'État de Rio Grande do Sul.

Les établissements montrent que les facteurs climatiques de la région sont les plus difficiles, tels que l'humidité, de fortes pluies, le gelés et la grêle. Ils montrent que ces facteurs limitent la production, principalement en ce qui concerne la production de raisins du type vitis vinifera.

Toutefois, l'établissement 1 , qui fabriquait du vin fin biologique, produisait leur raisin dans la région de la «Campanha Gaúcha ». Dans cet établissement la production de raisin n'était pas un facteur limitant, alliant à la rencontre de cela que l'établissement a rapporté, que la région de la «Campanha Gaúcha » a un bon terroir pour le vin fin biologique.

Pour l'établissement 1 , les ressources financières ont été la principale limitation, puisque l'investissement dans l'usine a été élevée, ainsi que le prix de la certification, car au moment de l'audit était nécessaire de prélever des échantillons vers des laboratoires d'ailleurs, ce qu'a augmenté le coût final du produit, au-delà de la valeur aperçue.

Les établissements 2, 3 e 5 ont dû former son assistance technique pour être en mesure de se rencontrer et apprendre à leurs fournisseurs pour produire les vignes biologiques et ont eu comme limitant la confiance dans les producteurs de raisins qui ont eu besoin d'être constamment surveillé, car il a encore des difficultés, par les restrictions que la production biologique présente.

Les établissements 2, 3, 4 et 5 montrent que l'organisme de contrôle et certification contribue à remédier à cette difficulté. D'autres institutions apportent également des contributions à la production et commercialisation, comme EMATER (Entreprise d'assistance technique et de développement rural de Rio Grande do Sul), qu'a donné l'assistance dans la conversion à la production biologique à l'établissement 1, SEBRAE (Service brésilien d'appui aux micro entreprises) qu'a donné de cours à l'établissement 4 pour gérer mieux la commercialisation de produits biologiques.

A l'EMBRAPA (Entreprise brésilienne de recherche agricole) a été citée par tous les établissements, elle développe des recherches des cépages plus résistantes, des techniques pour développer cette production et encore d'essais au champ dans les nouvelles frontières.

Les établissements 1, 2, 3 et 4 montrent la situation sanitaire des matières premières dont la grande limitation, puisque le facteur climatique et la non-utilisation de produits agrochimiques provoquent la pourriture dans les raisins, aussi que la situation sanitaire compromise par d'autres facteurs. Le risque encouru fait que de nombreux fournisseurs abandonnent la production de raisins biologiques.

\subsection{Fabrication}

Dans la fabrication, seulement l'établissement 1 a montré les limitations. En ayant une petite usine de production, il a fallu adapter les réservoirs et aussi l'usine. Cependant, les autres vignobles ont signalé que leur structure pour la production conventionnelle était déjà consolidée, cela a facilité ce processus.

Pour eux, lorsque les raisins arrivent, seulement ils doivent être traités dans différents moments que les conventionnelles. Typiquement, les raisins biologiques sont reçus et traités au début de la matinée, pour ensuite traiter les conventionnelles, pour n'avoir aucun contact entre eux autant que risques de contamination.

N'étant pas un facteur limitant, mais les soins nécessaires. Il est nécessaire de stocker et séparer le produit dans l'usine, outre d'identifier les produits et aussi garder l'équipement aseptisé, qui est une exigence sanitaire pour tous les produits.

Les difficultés dans la formation et le partage de l'industrie entre conventionnelle et biologique, ont fait l'établissement 2 à arrêter de produire, puisque l'attention n'est pas la production biologique.

\subsection{Commercialisation, distribuition et consommation}

Tous les établissements ont montré que le produit biologique est très important pour leur marque institutionnelle. En commençant par le vin, bien que ne 
sont pas négociés dans les grands réseaux, les produits font partie du portefeuille de la société, ainsi que leurs positionnements. L'établissement 1 , au-delà d'arrêter de produire et commercialiser les produits biologiques, montre toujours son souci de l'environnement dans la production de conventionnelle.

L'établissement 2, lors qu'il s'est rendu compte que les produits biologiques n'étaient pas l'objet de la société et aussi à cause des difficultés, il s'est concentré sur les produits conventionnelles, en laissant cette année la commercialisation des biologique.

Les établissements, sauf le 1, disent que le produit a encore peu d'importance sur le marché, car le type de vin biologique produit par eux est un produit peu apprécié dans le reste du pays, car le Brésil n'est pas assez un consommateur traditionnel de vin, donc les consommateurs cherchent par la qualité du produit [14].

Les établissements 3 , 4 et 5 montrent que le vin représente très peu de leurs revenus, ils le produisent pour avoir dans le portefeuille de produits et satisfaire le marché régional, puisque le but de la production biologique se concentre sur la production biologique de jus de raisin, qui est commercialisé dans tout le Brésil.

La distribution n'est pas un facteur limitant, puisque les produits après être emballés, doivent qu'être suivi et contrôlés, en suivant les mêmes procédures de distributions conventionnelles, même pas d'empaquetage et des moyens de transport différent ou qui s'approche à la durabilité. Le prix est la limitation la plus visible dans la commercialisation, ce qui, en raison des difficultés rencontrées dans la production de raisins biologiques, conduit à une augmentation significative du prix par rapport au produit conventionnel.

En outre, selon la loi 10.831 , qui régit la production biologique au Brésil [9], les produits doivent être exposés dans des endroits spécifiques et marqués, ce qui n'arrive pas toujours dans les supermarchés, qui sont également à proximité des conventionnelles, ce qui peut conduire les consommateurs à confusion.

La limitation majeure à cet égard est de fournir des vins fins aux consommateurs, en plus d'établissements vinicoles se concentrent sur la production de jus de raisin. Selon les établissements vinicoles, les consommateurs ne savent toujours pas le sens réel du produit, l'amenant à ne pas payer plus pour cela.

\subsection{Partie prenante et l'avenir}

Les parties prenantes sont des importants facteurs pour l'avenir de la production de vin biologique au Brésil. Les principaux parties prenantes cité par les établissements vinicoles étaient EMBRAPA, qui cherche des nouvelles façons de produire les raisins biologiques au Brésil, encore pour développer des variétés (cultivars) résistantes au climat. Cité par l'établissement 4, le SEBRAE a aussi eu un rôle important, principalement dans le processus de commercialisation.

En ce qui concerne à la commercialisation et l'organisation du secteur, ainsi que dans la production conventionnelle, l'IBRAVIN (Institut brésilien du vin et de la vigne) a un rôle important dans l'avenir et est une partie prenante importante, puisqu'ils ont commencé avec des campagnes publicitaires et actions marketing pour promouvoir le vin brésilien et le jus, mais c'est visible que manque des efforts pour les produits biologiques, puisque les établissements ont reporté que dans le système de contrôle de la production vinicole brésilien, où se déclare toute la production nationale de vin et jus de raisin, il n'y a pas une séparation entre les produits conventionnel et celui biologique.

Les établissements ont montré qu'elles ont été mieux vues par la communauté à cause de la production biologique, à cause des avantages environnementales et sociales que la production biologique apporte.

Quant à la production future, beaucoup d'établissements se sont déjà positionné pour production de jus de raisin biologique, car les cépages cultivés aujourd'hui ont de bonnes caractéristiques gustatives au jus. D'autre part, l'établissement 4 a rapporté que, donnée les caractéristiques de la région de la «Campanha Gaúcha », ce serait une alternative à la Région de la « Serra Gaucha » pour la production de raisins de la variété vitis vinifera et dans l'avenir, les établissements tend à produire d'autres produits vinicoles biologique.

L'établissement 5 a également signalé qu'envisage augmenter leurs produits biologiques offerts par l'eux, comme il l'a déjà intégré dans ses propres produits soustraités du point de vente.

L'établissement 1 a arrêté de certifier en raison du coût. L'établissement 2 n'ayant plus de production de vin biologique depuis quelque temps, commercialisait avec son étiquette, le produit était sous-traité d'autre producteur de la région. Celui-là a continué uniquement avec du jus de raisin biologique, qui a également retiré son portefeuille de produits aussi que le vin de table biologique.

Cependant, les établissements vinicoles croient qu'ils devraient y envisager des alternatives pour produire de tels produits, puisque la tendance de la consommation des produits biologiques augmente de plus en plus, selon eux, mais des difficultés, en particulier dans la production de raisins biologiques, doivent être pris en compte.

\section{Discussion}

Il est notable que les établissements sont devant à une difficulté d'un climat favorable à la production de raisins. Cela est déjà une question en recherche d'alternatives non seulement par l'EMBRAPA, mais aussi par d'autres établissements vinicoles qui ont migré vers les régions moins humides avec moins de problèmes climatiques, comme la région de la « Campanha Gaúcha».

D'autres régions pourraient également présenter des solutions possibles aux limitations actuelles, il est nécessaire de connaître la culture, en tant que régions sèches, comme la région centrale du Brésil, qui a un climat sec, mais par contre chaud. Un autre point qui a été trouvé comme solution aux vignobles, est de produire du vin de table biologique (variétés américaine) pour la région sud du Brésil, qui valorise le produit et le jus de raisin biologique à grande échelle (national), vu que le jus de raisin est très apprécié, même que dans certains cas les consommateurs ne parviennent pas à différer les significations entre celui conventionnel et biologique [14].

De toute façon, il est possible de conclure que même ayant une préoccupation de s'adapter à la certification, d'avoir de raisin de qualité et aussi de fournisseurs, il y a encore des efforts de marketing, de sorte que la valeur du produit atteint le consommateur final, car les vignobles 
reportent qu'il ne les faut plus d'effort pour faire que le produit soit connu et valorisé par le consommateur.

Ainsi, les vignobles pourraient inclure de nouvelles solutions qui ajoutent plus de valeur au produit, tel que la distribution et la commercialisation, l'utilisation des ressources renouvelables, les approches de marketing pour mieux transmettre cette valeur au consommateur final et des parties prenantes. De cette façon, la valeur agrée dans le système de valeur serait plutôt pour toute l'image de l'entreprise, comme une valeur de durabilité.

De plus, il est possible d'identifier que le produit apporte une valeur ajoutée à d'autres produits et qu'il faut encore des efforts pour trouver des nouvelles technologies pour la production de variétés vitis vinifera, puisque, jusqu'au présent, les vins biologiques sont produits qu'à partir de variétés américaines.

Enfin, il est possible de noter l'importance des institutions comme le SEBRAE, l'EMBRAPA et l'IBRAVIN pour le développement et l'avenir de cette production, autant que l'importance du jus de raisin dans cette chaine productive, qui a démontré un agrandissement de la production et de la demande de la part du consommateur. Ces défis ont fait que les établissements concentrent leurs productions dans le jus.

\section{Références}

[1] MINTEL, "Global Food \& Drink Trends 2016," 2015

[2] E. Ulusoy, "Experiential responsible consumption," J. Bus. Res., vol. 69, pp. 284-297, 2016

[3] F. PORTILHO, Sustentabilidade ambiental, consumo e cidadania, 1st ed. São Paulo: Cortez, 2005

[4] J. C. Nachtigal, M. Botton, H. P. dos Santos, L. da R. Garrido, F. Hillebrand, G. Onsi, and V. Bellé, "Recomendações para produção de uvas de mesa em cultivo protegido na região da Serra Gaúcha," Documentos. Embrapa Uva e Vinho, Bento Gonçalves, p. 29, 2010

[5] L. M. R. de Mello and J. F. da S. Protas, "Uvas Viníferas para Processamento em Regiões de Clima Temperado," Sistema de Produção, 4, 2003. [Online].
Available: https://sistemasdeproducao. cnptia.embrapa.br/FontesHTML/Uva/ UvasViniferasRegioesClimaTemperado/ mercado.htm\#topo. [Accessed: 20-Jan-2016]

[6] Planeta-Orgânico, "O mundo dos vinhos orgânicos," Planeta Orgânico, 2003 [Online]. Available: http://planetaorganico.com.br/site/index. php/o-mundo-dos-vinhos-organicos-2/. [Accessed: 25-Jun-2015]

[7] H. J. Lee and Z. S. Yun, “Consumers' perceptions of organic food attributes and cognitive and affective attitudes as determinants of their purchase intentions toward organic food," Food Qual. Prefer., vol. 39, pp. 259-267, 2015

[8] C. Hempel and U. Hamm, "How important is local food to organic-minded consumers?," Appetite, vol. 96, pp. 309-318, 2016

[9] BRASIL, "LEI N. 10.831 - Agricultura orgânica," 2003. [Online]. Available: http://www . planalto . gov.br/ccivil_03/leis/2003/L10.831.htm. [Accessed: 04-Sep-2016]

[10] M. E. Porter, Vantagem competitiva - Criando e sustentando um desempenho superior. Rio de Janeiro: CAMPUS, 1989

[11] C. C. Poirier and S. E. Reiter, Supply chain optimization: building the strongest total business network, 1st ed. San Francisco: Berrett-Koehler, 1996

[12] P. Kotler, H. Kartajaya, and I. Setiawan, Marketing 3.0: As forças que estão definindo o novo marketing centrado no ser humano. Rio de Janeiro: Elsevier, 2010

[13] FIESP-IBOPE, "Brasil Food Trends 2020," 2010. [Online]. Available: http://www.abic. com.br/media/EST_PESQFoodTrendsl.pdf . [Accessed: 10-May-2015]

[14] L. M. R. De Mello, "Tendência de Consumo e Perspectivas do Mercado de Vinhos no Brasil." Embrapa Uva e Vinho, Bento Gonçalves, p. 7, 2002 